msh-mss Mathématiques et sciences humaines

157 | Printemps 2002

Varia

\title{
Graphes d'arches
}

Arch graphs

Bruno Leclerc

\section{(2) OpenEdition}

Journals

Édition électronique

URL : http://journals.openedition.org/msh/2858

DOI : $10.4000 / \mathrm{msh} .2858$

ISSN : 1950-6821

\section{Éditeur}

Centre d'analyse et de mathématique sociales de l'EHESS

Édition imprimée

Date de publication : 1 mars 2002

ISSN : 0987-6936

\section{Référence électronique}

Bruno Leclerc, "Graphes d'arches », Mathématiques et sciences humaines [En ligne], 157 | Printemps 2002, mis en ligne le 10 février 2006, consulté le 23 juillet 2020. URL : http://journals.openedition.org/ msh/2858; DOl : https://doi.org/10.4000/msh.2858 


\title{
GRAPHES D'ARCHES
}

\author{
Bruno LECLERC ${ }^{1}$
}

\begin{abstract}
RÉSUMÉ - Un graphe d'arches s'obtient à partir d'une simple arête par ajouts successifs de 3chaînes, greffées sur leurs extrémités. De façon équivalente, c'est un graphe sans sous-graphe dont tous les sommets sont de degré au moins trois et maximal avec cette propriété à nombre de sommets fixé. Il est connu qu'une distance d'arbre est résumable par $2 n-3$ de ses entrées, bien choisies. Les graphes d'arches à $n$ sommets correspondent à de tels ensembles d'entrées. Ils contiennent la sous-classe bien étudiée des 2-arbres. Nous étudions ces graphes, et les graphes de $k$-arches et $k$-arbres qui les généralisent naturellement. Nous rappelons comment on passe d'un graphe d'arches valué à une fonction ou une distance d'arbre et nous examinons les propriétés de cette correspondance.
\end{abstract}

MOTS-CLÉS - Arbre, 2-arbre, Cycle, Distance, Graphe, Algorithme, Codage d'arbre

SUMMARY - Arch graphs

An arch-graph may be obtained from a simple edge by successive addings of 3-paths, grafted on their extremities. Equivalently, it admits no subgraph of which every vertex has degree at least three, and is maximal with this property, for a fixed number of vertices. It is known that a tree distance may be summarized by $2 n-3$ of its entries, conveniently chosen. Arch graphs with $n$ vertices correspond to such sets of entries. They include the graphs of the so-called 2-tree type. We study these graphs, and the $k$ arch graphs and k-trees which naturally generalize them. It is recalled how a tree metric or function is associated to a valued arch graph, and the properties of this correspondence are investigated.

KEYWORDS - Tree, 2-tree, Cycle, Distance, Graph, Algorithm, Tree encoding

\section{INTRODUCTION}

Dans cet article essentiellement bibliographique, nous présentons une classe de graphes, appelés, pour des raisons indiquées au paragraphe 3, graphes d'arches. Ces graphes sont, semble-t-il, peu apparus dans la littérature, en dehors de [48] et de leur sous-classe bien étudiée (depuis Harary et Palmer [27]) des $k$-arbres. Nous avons d'abord rencontré cette dernière à propos de problèmes de codage d'arbres, de classification et d'analyse des dissimilarités [42], [43], [38]. Au cours de travaux en commun [25], [26], Alain Guénoche nous a fait remarquer que les graphes d'arches constituaient en fait la classe de graphes à considérer.

\footnotetext{
${ }^{1}$ Centre d'Analyse et de Mathématique Sociales, École des hautes études en sciences sociales, 54 bd Raspail 75270 Paris cedex 06, leclerc@ehess.fr
} 
On pourra consulter le livre de Barthélemy et Guénoche [6] sur la plupart des définitions et résultats suivants : soit $X$ un ensemble fini à $n$ éléments. Une dissimilarité sur $X$ est une fonction réelle $d \operatorname{sur} X \square X$ vérifiant $d(x, y)=d(y, x)$ et $d(x, y) \geq d(x, x)=0$ pour tous $x, y \square X$. C'est une distance si, pour tous $x, y, z \square X, d(x, z) \leq d(x, y)+d(y, z)$. C'est une distance d'arbre si, pour tous $x, y, z, w \square X$, l'inégalité suivante (F) est satisfaite :

$$
d(x, y)+d(z, w) \leq \max \{d(x, z)+d(y, w), d(x, w)+d(y, z)\}
$$

Un arbre non orienté dont les $n$ feuilles sont étiquetées selon $X$ et les autres sommets (appelés sommets virtuels) sont de degré au moins 3 est appelé dans la suite un FEX-arbre. Les arêtes incidentes aux feuilles y sont dites extérieures, et celles entre deux sommets virtuels sont dites intérieures. On dit qu'un tel arbre est complètement résolu si tous ses sommets virtuels sont de degré 3 ; il a alors $2 n-3$ arêtes. On sait qu'une distance d'arbre sur $X$ est représentable, de façon unique, par les longueurs des chemins entre les feuilles d'un FEX-arbre [14]. Une extension de ce résultat s'obtient en restreignant (F) aux quadruplets d'éléments distincts de $X$ [36]. Une dissimilarité (resp. une fonction réelle sur $X \square X)$ vérifiant cette dernière propriété est appelée une dissimilarité d'arbre (resp. une fonction d'arbre). Une telle fonction $d$ se transforme aisément en une distance d'arbre $d$ ' en ajoutant une constante positive suffisante $2 c$ à chacune de ses entrées. Soit alors le FEX-arbre $T$ correspondant à $d$ '. En diminuant de $c$ les longueurs des arêtes extérieures de $T$ (ce qui peut rendre négatives certaines de ces " longueurs"), on retrouve une représentation de $d$ par un FEX-arbre. On en déduit qu'une fonction d'arbre a une unique représentation par un FEX-arbre, avec éventuellement des longueurs négatives sur certaines arêtes extérieures.

Une classe particulière de distances d'arbres est constituée par les ultramétriques, qui vérifient $d(x, z) \leq \max \{d(x, y), d(y, z)\}$ pour tous $x, y, z \square X$. On sait qu'une ultramétrique est parfaitement définie par sa restriction aux $n$-1 entrées correspondant aux arêtes de l'un de ses arbres minimums (voir par exemple [34], [37]). Par ailleurs, on sait aussi trouver des ensembles de $2 n$-3 entrées résumant une distance d'arbre ([16], [52], puis [35], [36], [42], [43], [38]), ce qui a été appliqué, notamment, à la reconstruction phylogénétique avec données manquantes [25], [26]. Ces entrées correspondent aux arêtes de graphes (aux sommets étiquetés selon $X$ ) d'un type particulier, celui des "graphes d'arches", et à une façon de décomposer ceux-ci en " arches ", chaque arche correspondant à la greffe d'une nouvelle feuille sur le FEXarbre courant.

Au paragraphe 2, nous présentons cette construction par laquelle on passe d'un graphe d'arches à un FEX-arbre, tous deux valués. Au paragraphe 3, nous décrivons les arbres généralisés que sont les graphes d'arches, ainsi que la classe particulière des 2arbres dont nous faisons une revue bibliographique au paragraphe 4. Au paragraphe 5, nous examinons les propriétés de la correspondance entre graphes d'arches et FEXarbres valués, en particulier celles d'unicité et d'inversibilité. 


\section{LA MÉTHODE DES TRIANGLES}

On suppose que l'on a un graphe $G=(X, E)$, où $E$ est l'ensemble des paires d'éléments de $X$ sur lesquelles la valeur de la fonction $d$ est donnée (ou connue). La donnée est donc le graphe $G$ valué par $d$ et on considère la situation particulière où le graphe $G$ vérifie la condition (OEI) suivante d'existence d'un "ordre d'élimination " (inversé) : il existe un ordre $x_{1}, x_{2}, \ldots, x_{\mathrm{n}}$ sur $X$ tel que $x_{1} x_{2} \square E$ et, pour tout $x_{i}, i \geq 3$, il existe exactement deux éléments $y, z \square\left\{x_{1}, \ldots, x_{\mathrm{i}-1}\right\}$ avec $x_{\mathrm{i}} y, x_{\mathrm{i}} z \square E$. La figure 1 montre un tel graphe avec l'ordre $\{a, b, c, d, e, f\}$ sur ses sommets.

On associe alors de façon non ambiguë un unique FE $X$-arbre $T$ positivement valué sur ses arêtes intérieures (on verra en section 5 qu'il ne dépend pas de l'ordre sur $X$ utilisé), en procédant comme suit :

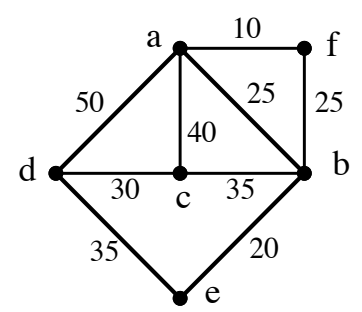

Figure 1

- Le triangle $\left\{x_{1}, x_{2}, x_{3}\right\}$ est d'abord représenté de façon unique par une 3-étoile $S_{3}$. Celle-ci est le $\operatorname{FE}\left\{x_{1}, x_{2}, x_{3}\right\}$-arbre valué admettant un unique sommet virtuel $u$ et trois arêtes $u x_{1}, u x_{2}$ et $u x_{3}$, toutes extérieures. Les longueurs $\ell\left(u x_{1}\right), \ell\left(u x_{2}\right)$ et $\ell\left(u x_{3}\right)$ de ces arêtes sont déterminées par les égalités $2 \ell\left(u x_{1}\right)=d\left(x_{1}, x_{2}\right)+d\left(x_{1}, x_{3}\right)-d\left(x_{2}, x_{3}\right)$, $2 \ell\left(u x_{2}\right)=d\left(x_{1}, x_{2}\right)+d\left(x_{2}, x_{3}\right)-d\left(x_{1}, x_{3}\right)$, et $2 \ell\left(u x_{3}\right)=d\left(x_{1}, x_{3}\right)+d\left(x_{2}, x_{3}\right)-d\left(x_{1}, x_{2}\right)$. Ces longueurs sont toutes non-négatives si et seulement si le triangle $\left\{x_{1}, x_{2}, x_{3}\right\}$ valué par $d$ est métrique.

- On procède de même pour le triangle $\left\{x_{4}, y, z\right\}$, où $y, z \square\left\{x_{1}, x_{2}, x_{3}\right\}$, ce qui donne une seconde 3-étoile $S_{4}$ possédant un chemin $S_{4}(y z)$ entre $y$ et $z$ en commun avec $T_{3}(y z)$, et avec la même longueur $d(y, z)$. On peut donc recoller les arbres $S_{3}$ et $S_{4}$ sur ce chemin pour obtenir un $\operatorname{FE}\left\{x_{1}, x_{2}, x_{3}, x_{4}\right\}$-arbre $T^{4}$. De plus, quitte à introduire des arêtes extérieures de longueurs négatives, ce recollement peut être fait de façon unique avec des arêtes intérieures positivement valuées [38].

- Pour chaque $i=5, \ldots, n$, un nouveau triplet de sommets $x_{i}, y$ et $z$ avec $y, z \square$ $\left\{x_{1}, x_{2}, \ldots, x_{\mathrm{i}-1}\right\}$ et $x_{i} y, x_{i} z \square E$, est considéré à la $i$-2-ième étape. Si $y z \square E$, le triplet $\left\{x_{i}, y, z\right\}$ constitue un triangle (3-clique) de $G$. Dans tous les cas, nous dirons que ce triplet est une arche de $G$ et, si $y z \square E$, nous nous ramenons au cas précédent en prenant pour valeur de $d(y, z)$ la longueur $d_{\mathrm{T}^{i-1}}(y, z)$ du chemin entre $y$ et $z$ dans le $\mathrm{FE}\left\{x_{1}, x_{2}, \ldots, x_{\mathrm{i}-1}\right\}$ - arbre courant $T^{i-1}$. Recoller sur ce chemin la 3étoile $S_{i}$ à l'arbre $T^{i-1}$ revient à greffer une nouvelle feuille $x_{i}$ sur $T^{i-1}$ pour obtenir le $\operatorname{FE}\left\{x_{1}, x_{2}, \ldots, x_{i}\right\}$-arbre $T^{i}$. Une observation, qui servira au paragraphe 5, est qu'à chaque étape, le triplet $\left(y, x_{i}, z\right)$ est bien formé dans $T^{i}$ au sens de [6], c'est-à-dire que l'arête extérieure incidente à la feuille $x_{i}$ a son autre extrémité placée sur le chemin de $T^{i}$ entre $y$ et $z$. 
On obtient finalement, à la $n$-2-ième étape, un FEX-arbre $T=T^{n}$ dont les longueurs de chemins préservent toutes les valeurs de $d$. Cette procédure en $O\left(n^{2}\right)$, illustrée par la figure 2, a été introduite dans [36], puis formalisée et étudiée dans [42], [38], [25], [26]. Au paragraphe suivant, nous étudions diverses classes de graphes, incluant ceux qui vérifient la condition (OEI).
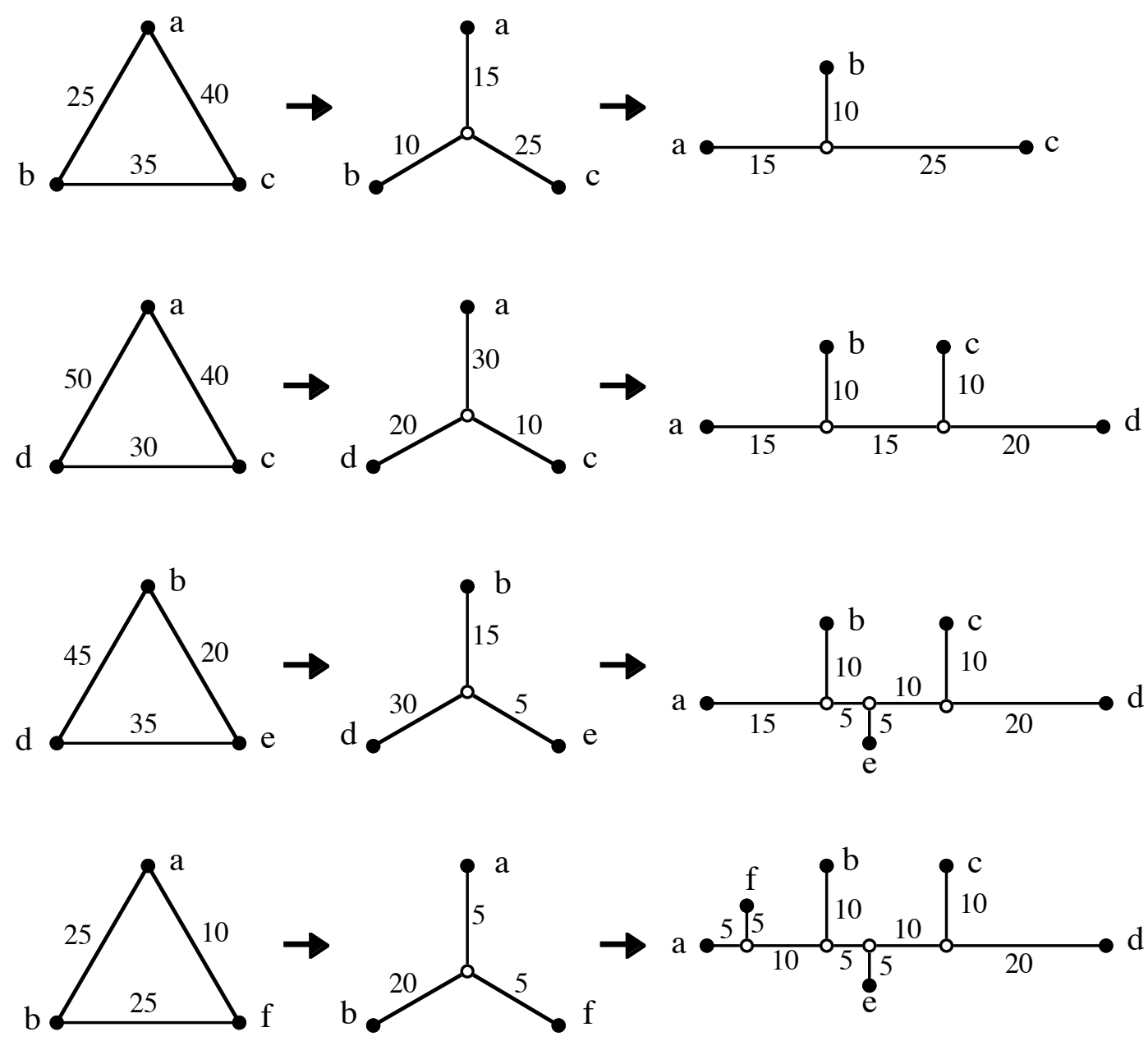

Figure 2

\section{ARBRES GÉNÉRALISÉS}

\section{$3.1 k \mathrm{~d}$-CYCLES ET GRAPHES D’ARCHES}

Soient $G=(X, E)$ un graphe (simple, non orienté, à $n$ sommets et $m$ arêtes) sur $X$, un ensemble d'arêtes $A \square E$, et l'ensemble $X_{A}$ des sommets de $G$ incidents à au moins une arête de $A$. On obtient un sous-graphe $G_{A}=\left(X_{A}, A\right)$ de $G$.

DÉFINITION 3.1 Une partie $C \square E$ est un $k \mathrm{~d}$-cycle (d pour degré) de $G$ si tous les sommets de $X_{C}$ sont incidents à au moins $k+1$ arêtes dans $G_{C}$ et si $C$ est minimale 
par l'inclusion avec cette propriété. Clairement, un 1d-cycle est un cycle au sens habituel. Nous nous intéresserons essentiellement à la valeur $k=2$.

- Si $C$ est un $k$ d-cycle et $x y \square C$, alors au moins un des sommets $x$ et $y$ est de degré exactement $k+1$ dans $G_{C}$.

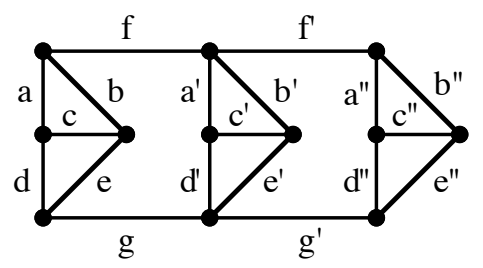

Figure 3

- Pour $k \geq 2$, l'ensemble des $k$ d-cycles de $G$ ne constitue pas en général l'ensemble des circuits d'un matroïde sur $E$, comme le montre le contre-exemple suivant. Dans le graphe de la figure 3, les ensembles d'arêtes $C=\left\{a, b, c, d, e, f, g, a^{\prime}, b^{\prime}, c^{\prime}, d^{\prime}, e^{\prime}\right\}$ et $C^{\prime}=\left\{a^{\prime}, b^{\prime}, c^{\prime}, d^{\prime}, e^{\prime}, f^{\prime}, g^{\prime}, a^{\prime \prime}, b^{\prime \prime}, c^{\prime \prime}, d^{\prime \prime}, e^{\prime \prime}\right\}$ sont des $2 d$-cycles tandis que le graphe $G_{(C \square C)-\{e\}}$ est sans 2 d-cycle, pour toute arête $e \square C \square C^{\prime}$. Les 2 d-cycles ne vérifient donc pas en général la propriété d'échange des circuits d'un matroïde (cf. par exemple [51]).

Un graphe sans $k \mathrm{~d}$-cycles est dit $k \mathrm{~d}$-acyclique. Les graphes $k \mathrm{~d}$-acycliques maximaux (à nombre de sommets $n$ fixé) sont appelés ici graphes de k-arches (et graphes d'arches pour $k=2$ ). Ils ont été caractérisés récursivement par Todd [48], comme étant exactements ceux pouvant s'obtenir de la façon suivante :

- Le graphe complet $K_{k}$ à $k$ sommets est un graphe de $k$-arches ;

- Si $G=(X, E)$ est un graphe de $k$-arches, alors, pour tout sous-ensemble $Y$ à $k$ éléments de $X$ et tout nouveau sommet $z \square X$, le graphe $G^{\prime}=(X \square\{z\}, E \square\{z y: y$ $\square Y\}$ ) est un graphe de $k$-arches.

C'est pour évoquer l'ancrage du nouveau sommet $z$ sur $k$ anciens sommets que nous proposons graphe de $k$-arches (" $k$-arch graph "). Avec cette construction, on voit qu'un graphe de $k$-arches à $n$ sommets est connexe, a exactement $\frac{k(2 n-k-1)}{2}$ arêtes, et a au moins un sommet de degré $k$. Todd [48] appelle “ $k$-trees ” $(k$-arbres $)$ ces graphes mais cette dénomination est inadéquate puisque déjà attribuée dans la littérature à des graphes satisfaisant des conditions supplémentaires, décrits au paragraphe suivant.
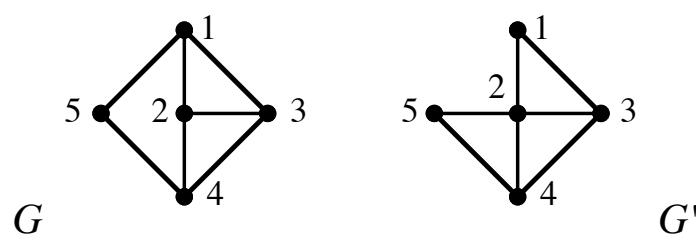

Figure 4

Pour $k=2$, un graphe d'arches à $n$ sommets a $2 n-3$ arêtes. De plus, un graphe $G=(X, E)$ est un graphe d'arches s'il existe un ordre total $\left(x_{1}, x_{2}, \ldots, x_{n}\right)$ sur $X$ tel que 
$x_{1} x_{2} \square E$ et, pour $i=3, \ldots, n$, le sommet $x_{i}$ est de degré 2 dans le sous-graphe induit $G_{i}=G\left\{x_{1}, x_{2}, \ldots, x_{i}\right\}$, c'est-à-dire que les graphes d'arches sont exactement les graphes vérifiant la condition (OEI) du paragraphe 2. Les graphes $G$ et $G^{\prime}$ de la figure 4 sont tous deux des graphes d'arches, ainsi que celui de la figure 1, mais non celui de la figure 3 , qui n'est pas 2d-acyclique.

Todd [48] propose un algorithme pour reconnaître si un graphe $G=(X, A)$ donné contient ou non un $k$ d-cycle. On construit un sous-ensemble $B$ de $A$, le k-grattage (le terme est introduit ici ; nous proposons $k d$-cleaning dans [26]) de $A$ comme suit :

- Si $G$ n'a pas de sommet de degré au plus $k$, alors $B=A$;

- Sinon, on enlève un sommet de degré au plus $k$, et les arêtes qui lui sont incidentes, dans $G$ et on recommence cette opération jusqu'à ce qu'il ne reste plus de tel sommet. L'ensemble des arêtes restantes est $B$.

Dans tous les cas, le graphe $G$ contient un $k$ d-cycle si et seulement si $B \neq \varnothing$.

\subsection{2-CYCLES ET 2-ARBRES}

Une classe particulière de graphes de $k$-arches correspond à une seconde généralisation des cycles et des arbres. Rappelons d'abord une définition classique de la théorie des graphes (cf. [27], ch. 11, p. 107) : une arête $x y$ d'un graphe $G$ peut être subdivisée en la remplaçant par deux arêtes $x z$ et $y z$ ( $z$ étant un sommet nouveau), et deux graphes $H$ et $H^{\prime}$ sont dits homéomorphes s'ils peuvent être obtenus à partir d'un même graphe $G$ par des suites de telles subdivisions. Ainsi, tout cycle est homéomorphe à un triangle.

DÉFINITION 3.2 Une partie $C \square E$ est un k-cycle de $G$ si le graphe $G_{C}$ est homéomorphe au graphe complet à $k+2$ sommets $K_{k+2}$. En particulier, un 1-cycle est un cycle et un graphe homéomorphe à $\mathrm{K}_{4}$ est un 2-cycle.

- Si $C$ est un $k$-cycle, un sommet $x$ de $G_{C}$ est de degré exactement 2 ou $k+1$ dans $G_{C}$.

- De nouveau, l'ensemble des 2-cycles d'un graphe $G$ donné ne constitue pas en général l'ensemble des circuits d'un matroïde sur $E$, comme le montre le contreexemple de la figure 5. Dans le graphe considéré, les ensembles $C=\{\mathrm{a}, \mathrm{b}, \mathrm{c}, \mathrm{d}, \mathrm{e}, \mathrm{f}$, $\mathrm{g}$ \} et $C^{\prime}=\left\{\mathrm{a}^{\prime}, \mathrm{b}^{\prime}, \mathrm{c}, \mathrm{d}, \mathrm{e}, \mathrm{f}, \mathrm{g}\right\}$ sont des 2-cycles mais, pour l'arête $\mathrm{d} \square C \square C^{\prime}$, le sous-graphe induit $G_{(C \square C) \text {-\{d\} }}$ est sans sous-graphe homéomorphe à $K_{4}$.

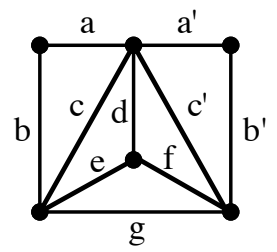

Figure 5

Un graphe sans $k$-cycles est dit $k$-acyclique. Les graphes $k$-acycliques maximaux sont appelés $k$-arbres. Ils ont été bien étudiés dans la littérature (voir le $\S 4$ ci-dessous). En particulier, leur caractérisation récursive montre qu'ils constituent des graphes de $k$ - 
arches particuliers; les $k$-arbres sont exactements les graphes pouvant s'obtenir de la façon suivante :

- Le graphe complet $K_{k}$ à $k$ sommets est un $k$-arbre ;

- Si $G=(X, E)$ est un $k$-arbre, alors, pour toute clique $Y$ à $k$ éléments de $G$ et tout nouveau sommet $z \square X$, le graphe $G^{\prime}=(X \square\{z\}, E \square\{z y: y \square Y\})$ est un $k$-arbre.

En d'autres termes, un graphe $G=(X, E)$ est un $k$-arbre si et seulement si il existe un ordre total $\left(x_{1}, x_{2}, \ldots, x_{n}\right)$ sur $X$ tel que $x_{1} x_{2} \ldots x_{k}$ est une clique et, pour $i=$ $k+1, \ldots, n$, le sommet $x_{i}$ est de degré $k$ et appartient à une unique $(k+1)$-clique du sous-graphe induit $G_{i}=G_{\left\{x_{1}, x_{2}, \ldots, x_{i}\right\}}$. Un $k$-arbre a au moins deux sommets de degré $k$.
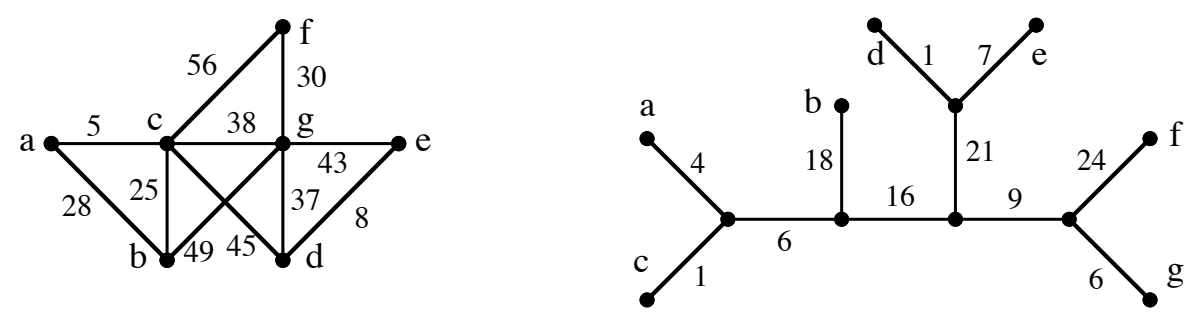

Figure 6

Le graphe $G$ ' de la figure 4 est un 2-arbre, alors que $G$ est un graphe d'arches qui n'est pas un 2-arbre (noter qu'il s'agit d'un 2-cycle). La figure 6 montre un 2-arbre valué (à gauche), ainsi que le FEX-arbre que l'on obtient en lui appliquant la méthode des triangles (à droite). Les 2-arbres ont été bien étudiés dans la littérature depuis Harary et Palmer [28], Moon [44], Pippert et Beineke [45], et ce d'autant qu'ils sont à la rencontre de plusieurs classes de graphes (graphes triangulés, série-parallèles,...) intéressantes en algorithmique, comme il sera rappelé aux paragraphes 4.2 et 4.3.

\subsection{UN LIEN ENTRE $k$ d-CYCLES ET $k$-CYCLES}

Une première application de ce qui précède est la démonstration très simple, comme conséquence directe des caractérisations récursives des graphes de $k$-arches et des $k$ arbres rappelées précédemment, de la propriété suivante :

PROPOSITION 3.1. Un kd-cycle n'est pas k-acyclique.

Preuve. Sinon, soit $C$ un $k$ d-cycle tel que $G_{C}$ ne contient pas de $k$-cycle. Alors, $G_{C}$ est un sous-graphe d'un graphe maximal $H$ sans $k$-cycle, c'est-à-dire d'un $k$-arbre. Mais $H$ est aussi un graphe de $k$-arches, ce qui contredit l'hypothèse selon laquelle $C$ est un $k \mathrm{~d}$-cycle.

Pour $k=2$, ce résultat est une variante d'un lemme bien connu de Dirac ([19] ; voir par exemple [51], p. 238, ou [1], p. 387), souvent selon un énoncé restreint aux graphes 2-connexes: un graphe dont tous les sommets sont de degré au moins 3 contient un sous-graphe homéomorphe à $K_{4}$. Une autre conséquence directe de la 
proposition 3.1 est qu'un graphe dont tous les sommets sont de degré 4 ne peut être planaire. Il contient en effet un sous-graphe homéomorphe à $K_{5}$ et l'on n'a plus qu'à appliquer le théorème de Kuratowski ([33] ; cf. [27], p. 109, [8], p. 20). La figure 7 montre un 2d-cycle $C$ non 2-connexe et contenant deux 2-cycles $\{\mathrm{a}, \mathrm{b}, \mathrm{c}, \mathrm{d}, \mathrm{e}, \mathrm{f}, \mathrm{g}\}$ et $\left\{a^{\prime}, b^{\prime}, c^{\prime}, d^{\prime}, e^{\prime}, f^{\prime}, g^{\prime}\right\}$. Noter que l'arête $h$ n'appartient à aucun 2-cycle de $C$.

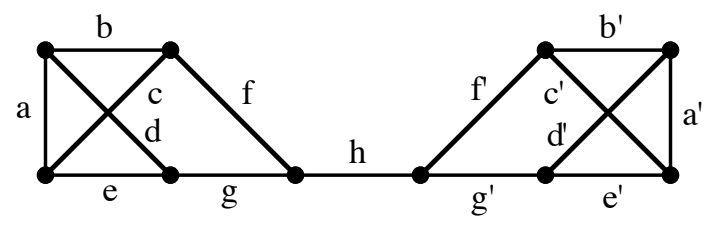

Figure 7

\section{PROPRIÉTÉS DES $k$-ARBRES}

On a dit plus haut que les $k$-arbres ont été bien étudiés dans la littérature. Ils correspondent en effet à une généralisation $k$-dimensionnelle naturelle des arbres et ils ont de nombreuses propriétés. Cette classe semble cependant être restée assez mal connue. La raison pourrait en être que l'intérêt s'est finalement porté sur des classes plus vastes (graphes triangulés, $k$-arbres partiels,...). Il peut donc être utile d'en faire une rapide revue bibliographique. On notera que les $k$-arbres sont liés à la classification de deux façons : à travers le problème de la phylogénie parfaite ( $\$ 4.5$ ci-dessous) et - avec les graphes d'arches - à propos du résumé d'une dissimilarité d'arbre par $2 n-3$ entrées (paragraphe 5).

\subsection{LES DÉBUTS : COMPLEXES SIMPLICIAUX ET GRAPHES}

Les 2-arbres sont définis par Harary et Palmer [27] comme 2-complexes simpliciaux purs acycliques. Un 2-complexe simplicial assemble des 0-simplexes (sommets), 1simplexes (arêtes) et 2-simplexes (triangles) comme un 1-simplexe (graphe) des sommets et des arêtes. Par " purs", on entend que tout $r$-simplexe, $r<2$, est inclus dans un 2-simplexe (triangle), ou, en d'autres termes, que tout sommet appartient (est incident) à une arête et toute arête à un triangle. Harary et Palmer définissent les 2arbres à partir des (1,2)-parcours : si $C$ est un 2-complexe pur, une suite $\ell_{0}, t_{1}, \ell_{1}, t_{2}, \ldots$, $t_{m-1}, \ell_{m-1}, t_{m}, \ell_{m}$ de simplexes de $C$ est un (1,2)-parcours si $\ell_{0}, \ell_{1}, \ldots, \ell_{m-1}, \ell_{m}$ sont des arêtes et $t_{1}, t_{2}, \ldots, t_{m-1}, t_{m}$ des triangles de $C$ et si le triangle $t_{i}$ est incident aux arêtes $\ell_{i-1}$ et $\ell_{i}$, pour $i=1, \ldots, m$. Un (1,2)-parcours est un (1,2)-cycle si $m>1$ et $\ell_{0}=\ell_{m}$. Le complexe $C$ est $(1,2)$-connexe s'il existe un $(1,2)$-parcours entre toute paire d'arêtes et est un 2-arbre s'il est connexe et n'admet pas de (1,2)-cycle.

Harary et Palmer s'intéressent aux centres (caractérisés ensuite par Proskurowski [46]), automorphismes et dénombrements des 2-arbres. Pippert et Beineke ([45]) reprennent la définition précédente des 2 -arbres pour les caractériser. 
THÉORÈME 4.1 [45]. Les quatre conditions suivantes sont équivalentes pour un 2complexe simplicial pur $C \grave{a} n$ sommets (0-simplexes):

(1) $C$ est un 2-arbre.

(2) $\quad C$ est $(1,2)$-connexe et a $2 n-3$ arêtes.

(3) $\quad C$ est $(1,2)$-connexe et a $n$-2 triangles.

(4) $\quad C$ n'a pas de $(1,2)$-cycle et a $2 n-3$ arêtes et $n-2$ triangles.

\subsection{CARACTÉRISATIONS ET PROPRIÉTÉS DE BASE}

Les auteurs précédents présentent les $k$-arbres comme $k$-complexes simpliciaux acycliques purs généralisant les 2-arbres. En fait, la définition des $k$-arbres en termes de complexes simpliciaux s'est rapidement effacée (à partir de [44]) devant une définition en termes de graphes, précisément ceux constitués des sommets et des arêtes des complexes précédents. Le passage de l'une à l'autre de ces notions est sans ambiguïté car, du fait de l'acyclicité, toute $k$-clique du graphe correspond à un $k$-simplexe du complexe. Les $k$-arbres sont dès lors définis le plus souvent de la façon récursive énoncée au paragraphe 3.2.

On a vu (§ 3.1 et 3.2) qu'un $k$-arbre à $n$ sommets a $\frac{k(2 n-k-1)}{2}$ arêtes et a au moins deux sommets de degré minimum possible $k$. De plus, le nombre de ses $(k+1)$-cliques est $n-k$.

Un $k$-arbre est un graphe triangulé (" triangulated", ou " chordal ", ou encore " rigid circuit", en anglais), c'est-à-dire que tout cycle de longueur supérieure à 3 y a une corde. Une propriété caractéristique des graphes triangulés est qu'ils sont les graphes à élimination parfaite (perfect elimination graphs). Les sommets d'un tel graphe $G$ peuvent être étiquetés de 1 à $n$ de sorte que, pour tout $i$, le sommet $i$ et ses sommets adjacents forment une clique $C_{i}$ du sous-graphe $G_{i}$ induit par les sommets $i, \ldots, n$ (l'ordre $1, \ldots, n$ est alors appelé un ordre d'élimination; son inverse a été considéré aux paragraphes 2 et 3). Rose ([47]) reprend la définition précédente des $k$ arbres et note qu'ils constituent la classe particulière des graphes à élimination parfaite pour lesquels $\left|C_{i}\right|=k+1$, pour $i=1, \ldots, n-k-1$ Par la suite, les $k$-arbres ne sont souvent mentionnés dans la littérature que comme graphes à élimination parfaite particuliers, comme dans [23], ch. 4, pp. 100-101, où ils font l'objet de cinq exercices; on notera, à la suite de Idury et Schäffer [29], que la propriété de planarité des 3-arbres que l'exercice 8 de [23] demande de prouver n'est pas vraie en général (ainsi, on obtient un 3 -arbre en remplaçant une des parties libres à 3 éléments du graphe biparti complet $K_{3,3}$ par une clique).

Pour $n>k+1$, un $k$-arbre avec exactement deux $k$-feuilles (sommets de degré $k$ ) est appelé un $k$-chemin, et un $k$-arbre avec un nombre de $k$-feuilles égal au maximum possible $n$ - $k$ une $k$-étoile. En enlevant toutes les $k$-feuilles d'un $k$-arbre $G$ qui n'est pas une $k$-étoile, on obtient un $k$-arbre $G^{\prime}$. Si $G^{\prime}$ est un $k$-chemin, on dit que $G$ est une $k$ - 
chenille [46]. Dans le cas des 2-arbres, un 2-chemin sera dit strict s'il n'a pas de sommet de degré supérieur à 4 , et roue brisée s'il a un sommet de degré $n-1$.

On trouvera en annexe les types de 2 -arbres (non étiquetés) pour $n$ compris entre 4 et 8 , ainsi que les types de graphes d'arches pour $n \leq 6$. Si $[p . q]$ y désigne le $p$-ième graphe à partir de la gauche de la $q$-ième ligne, alors [1.1], [1.2], [2.1], [4.1] et [7.1] sont des 2-chemins stricts, [1.1], [1.2], [2.2], [4.3] et [8.1] sont des roues brisées, [4.2] et [7.2] sont des 2-chemins n'appartenant pas aux deux types précédents, [1.1], [1.3], [2.5], [6.4] et [9.5] sont des 2-étoiles. Les graphes d'arches qui ne sont pas des 2-arbres sont [1.4] et ceux de la troisième ligne. Un 2-arbre qui n'est pas une 2-chenille est [11.4] (un tel graphe a au moins 8 sommets).

Beineke et Pippert, d'une part, et Moon, d'autre part, ont dénombré les $k$-arbres étiquetés :

THÉORÈME 4.2 [7], [44]. Le nombre $a_{k}(n)$ des $k$-arbres étiquetés à $n$ sommets est :

$$
\left.a_{k}(n)=H_{k}^{n}\right]^{n}\left(k n-k_{2}+1\right)^{n-k-2}
$$

En particulier, $a_{1}(n)=n^{n-2}$ (formule de Cayley), tandis que le nombre des 2-arbres à $n$ sommets est $a_{2}(n)=H_{2}^{n}\left[(2 n-3)^{n-4}\right.$.

On trouve une revue des références sur le dénombrement des 2-arbres, avec une série de résultats nouveaux, notamment sur le cas non étiqueté, dans Fowler et al. [21].

On sait que les arbres se caractérisent de nombreuses façons. La recherche de résultats analogues pour les 2 -arbres a conduit à plusieurs résultats. Soient $x$ et $y$ deux sommets appartenant à la même composante connexe d'un graphe $G=(X, E)$. Une partie $S$ des sommets de $G$ est un $(x, y)$-séparateur si $x$ et $y$ n'appartiennent plus à la même composante connexe du sous-graphe induit par $X-S$. Considérons les conditions suivantes :

(i) le graphe $G$ est connexe ;

(ii) le graphe $G$ a au moins une $k$-clique et n'a pas de $(k+2)$-clique ;

(iii) Tout $(x, y)$-séparateur minimal de $G$ est une $k$-clique ;

(iv) Tout $(x, y)$-séparateur minimal de $G$ est une clique ;

(v) $\quad|E|=\frac{k(2 n-k-1)}{2}$;

(vi) Pour tous $x, y \square X$, non adjacents dans $G$, il existe exactement $k$ chemins entre $x$ et $y$ deux à deux sans autre sommet commun que $x$ et $y$.

THÉORÈME 4.3 [47]. Les cinq conditions suivantes sont équivalentes pour un graphe $G$ :

(5) $\quad G$ est un $k$-arbre ;

(6) $G$ vérifie les conditions (i), (ii) et (iii); 
(8) $G$ vérifie les conditions (i), (iii) et (v);

(9) $G$ vérifie les conditions (ii), (iv) et (vi).

Dans la même direction que la propriété (vi), un graphe est dit IFI (isolated failure immune) si la délétion de deux éléments, sommets ou arêtes, non adjacents ou incidents, le laisse connexe (cette propriété est notamment intéressante dans l'étude des réseaux ou des circuits électroniques). Farley [20] signale que les 2-arbres vérifient cette propriété, et Wald et Colbourn [50] montrent que ce sont les graphes IFI minimaux.

Pour généraliser les $(1,2)$-parcours du $\S 4.1$ ci-dessus, appelons $(k, k+1)$-parcours $(k<n)$ d'un graphe $G$ à $n$ sommets une suite $\ell_{0}, t_{1}, \ell_{1}, t_{2}, \ldots, t_{m-1}, \ell_{m-1}, t_{m}, \ell_{m}$ où $\ell_{0}$, $\ell_{1}, \ldots, \ell_{m-1}, \ell_{m}$ sont des $k$-cliques et $t_{1}, t_{2}, \ldots, t_{m-1}, t_{m}$ des $(k+1)$-cliques de $G$, la clique $t_{i}$ contenant $\ell_{i-1}$ et $\ell_{i}$, pour $i=1, \ldots, m$. Proskurowski [46] montre qu'à toute paire de sommets $x, y$ non adjacents d'un $k$-arbre $G$ correspond un $(k, k+1)$-parcours unique tel que :

- les cliques $\ell_{0}, \ell_{1}, \ldots, \ell_{m-1}, \ell_{m}$ sont les $x, y$ séparateurs minimaux de $G$;

- $x$ est adjacent à tous les éléments de $\ell_{0}$;

- $y$ est adjacent à tous les éléments de $\ell_{m}$.

Alors, le sous-graphe $G[x y]$ de $G$ induit par $\{x, y\} \square\left(\square_{0 \leq i \leq m} \ell_{i}\right)$ est un $k$-arbre dont $x$ et $y$ sont les seuls sommets de degré minimal $k$ (dits $k$-feuilles). Ce $k$-arbre est appelé le $k$-chemin de $G$ entre $x$ et $y$ [38]. Proskurowski [46] montre que la réunion des $(x, y)$-séparateurs minimaux de $G$ est une $(k-1)$-chenille, c'est-à-dire qu'il reste un ( $k$-1)-chemin lorsqu'on lui enlève ses $k$-feuilles. Il définit une distance $\square$ sur les sommets de $G$ liée aux $k$-chemins. Un $k$-cable entre $x$ et $y$ (non adjacents) est la réunion de $k$ chemins disjoints entre $x$ et $y$ (on trouve toujours un tel ensemble de chemins dans un $k$-arbre), et $\square(x, y)$ est la somme minimale des longueurs des chemins d'un $k$-cable. Dans le cas des 2-arbres, la distance $\square(x, y)$ entre $x$ et $y$ correspond au nombre de triangles du 2-chemin $G[x y]$, augmenté de 2. On trouve dans [46] un algorithme de détermination de $G[x y]$ pour deux sommets $x$ et $y$ non adjacents d'un $k$-arbre $G$.

\subsection{Classes VOISINES ET GÉNÉRALISATIONS}

Une généralisation des $k$-arbres a été proposée par Dewdney [18]. Un $(p, q)$-parcours $(p<q \leq k+1)$ d'un $k$-complexe pur $C$ est une suite $\ell_{0}, t_{1}, \ell_{1}, t_{2}, \ldots, t_{m-1}, \ell_{m-1}, t_{m}, \ell_{m}$, où $\ell_{0}, \ell_{1}, \ldots, \ell_{m-1}, \ell_{m}$ sont des $p$-simplexes et $t_{1}, t_{2}, \ldots, t_{m-1}, t_{m}$ des $q$-simplexes, le simplexe $t_{i}$ étant incident à $\ell_{i-1}$ et à $\ell_{i}$, pour $i=1, \ldots, m$. Un $(p, q)$-parcours est un $(p, q)$-cycle si $m>1$ et $\ell_{0}=\ell_{m}$. Le complexe $C$ est un $(p, q)$-arbre s'il est $(p, q)$-connexe et n'admet pas de $(p, q)$-cycle. Alors, les $(k-1, k)$-arbres correspondent à la définition des $k$-arbres précédents en termes de complexes.

Bondy [10] définit un triarbre (" tritree ") d'un graphe $G$ connexe comme un arbre de $G$ tel que les cycles fondamentaux qui lui sont associés sont tous des triangles. Un 
trigraphe est un graphe qui admet un triarbre. La classe des trigraphes recoupe largement celle des 2-arbres (en particulier, les 2-chemins et les 2-étoiles sont des trigraphes), mais aucune des deux n'inclut l'autre ; ainsi, pour $n>3$, le graphe complet $K_{n}$ est un trigraphe (dont toute étoile est un triarbre), qui n'est évidemment pas un 2arbre, tandis que le graphe de la figure 8 est un 2-arbre qui n'est pas un trigraphe.

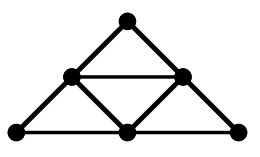

Figure 8

\subsection{GRAPHES $k$-ACYCLIQUES ( $k$-arbres partiels)}

À partir des années 80, l'intérêt se porte sur les $k$-arbres partiels, c'est-à-dire les graphes $k$-acycliques au sens du $\S 3.2$ ci-dessus. Ceci est dû à la reconnaissance du fait que plusieurs problèmes classiques et reconnus comme $N P$-difficiles ont des solutions polynomiales dans les graphes de ce type.

Wald et Colbourn [50] donnent la caractérisation des 2-arbres partiels en termes de configuration exclue, comme étant les graphes sans sous-graphe partiel homéomorphe à $K_{4}$; en d'autres termes, comme le notent Borie, Parker et Tovey [11], les 2-arbres partiels sont exactement les graphes série-parallèles. Wald et Colbourn donnent un algorithme en $O(n)$ pour résoudre le problème de Steiner (trouver un sousgraphe connexe minimal joignant un ensemble donné de sommets) dans un tel graphe. Les 2-arbres sont aussi les graphes triangulés maximaux sans 4-clique [47] ; sur ces classes de graphes, voir par exemple [23] ou [40]).

Les $k$-arbres partiels apparaissent alors, pour $k$ fixé, comme des généralisations des graphes série-parallèles (tandis que tout graphe est un $k$-arbre partiel pour un certain entier $k$ minimum à déterminer) : ce sont les graphes de largeur arborescente (treewidth) $k$ (cf. [32], [9]). Décrivons brièvement ce paramètre de largeur arborescente, dont l'importance en théorie algorithmique des graphes est due à ce que beaucoup de problèmes $N P$-difficiles en général deviennent polynomiaux, ou même linéaires, dans les graphes de largeur en arbre bornée (Hamiltonicité, $p$-colorabilité, cardinal maximum d'un stable, cardinal minimum d'une partie absorbante, planarité,...) [5] ; cf. [49]. Courcelle [17] a montré que tout problème exprimable en "logique monadique du second ordre " peut être - théoriquement - résolu en temps linéaire dans ces graphes.

Soit un graphe $G=(X, E)$. Une décomposition arborescente de $G$ est un couple $(\mathcal{X}$, $A$ ), où $X=\left\{X_{i}: i \square I\right\}$ est un ensemble de parties de $X$, indexées selon les sommets d'un arbre $A$, avec les propriétés : (i) $\square_{i \square I} X_{i}=X$; (ii) pour toute arête $x y \square E$, il existe $i \square I$ tel que $x, y \square X_{i}$; (iii) pour tous $i, j, k \square I$, si $j$ est sur le chemin de $A$ entre $i$ et $k$, alors $X_{i} \square X_{k} \square X_{j}$. La largeur de la décomposition $(\mathcal{X}, A)$ est le cardinal maximum d'un élément de $X$ et la largeur arborescente de $G$ est le minimum de cette largeur parmi toutes les décompositions arborescentes de $G$. C'est aussi le minimum 
du cardinal maximum d'une clique d'un graphe triangulé admettant $G$ comme sousgraphe partiel.

La reconnaissance des $k$-arbres partiels (ou graphes de largeur arborescente au plus $k)$ n'est pas en général un problème facile. Un algorithme en $O(\max (m, n))$ pour décider si un graphe donné $G$ à $m$ arêtes est ou non 2-acyclique a été donné par Liu et Geldmacher [39], et Arnborg et Proskurowski [3] [4] ont donné un algorithme en $O\left(n^{3}\right)$ pour la reconnaissance d'un 3-arbre partiel et sa complétion en un 3-arbre. Ensuite, Arnborg, Corneil et Proskurowski [2] étudient le même problème pour les $k$-arbres et montrent qu'il devient $N P$-difficile pour $k>3$ (une synthèse sur ce problème est faite dans [9]) ; il en est donc de même de la détermination de la largeur arborescente d'un graphe donné. D'autres classes de graphes pour lesquelles la largeur en arbre se détermine en temps polynomial sont reconnues dans Bouchitté et Todinca [12]. Granot et Skorin-Kapoff [24] proposent, après d'autres auteurs, des algorithmes parallèles pour la reconnaissance des 2 -arbres et des 3-arbres partiels.

Les $k$-arbres partiels ont été notamment utilisés dans la résolution de problèmes de satisfaction de contraintes (voir par exemple Freuder [22]).

\subsection{TRIANGULATION D'UN GRAPHE COLORÉ}

Étant donné un graphe ayant une coloration en $c$ couleurs, est-il possible de lui ajouter des arêtes de façon compatible avec la coloration (aucune nouvelle arête ne relie deux sommets de même couleur) jusqu'à l'obtention d'un graphe triangulé ? McMorris, Warnow et Wimer [41] montrent que ceci est possible si et seulement si le graphe triangulé obtenu est un $(c-1)$-arbre. Ils donnent un algorithme en $O\left((n+(c-1) m)^{c+1}\right)$, où $m$ est le nombre d'arêtes du graphe de départ, pour résoudre ce problème pour $c, m$ fixés (le problème général est $N P$-complet). Pour $c=3$ (le graphe solution devant donc être un 2-arbre), Kannan et Warnow [31] puis Idury et Schäffer [29] donnent des algorithmes performants.

Depuis Buneman [15], on sait que ce problème de triangulation est polynomialement équivalent au problème de la phylogénie parfaite (perfect phylogeny problem), qui se décrit ainsi : étant donné un ensemble $F$ d'espèces décrites par des caractères (chacun pouvant prendre plusieurs états), trouver un arbre dont les feuilles sont étiquetées selon $F$ et où chaque état d'un caractère correspond à un sous-arbre.

\section{PROPRIÉTÉS DE LA CORRESPONDANCE ENTRE GRAPHES D'ARCHES SUR $X$ ET FE $X$-ARBRES}

La méthode des triangles, décrite ci-dessus au paragraphe 2, associe un FE $X$-arbre, positivement valué sur les arêtes intérieures, à tout graphe d'arches valué, d'où une fonction d'arbre préservant les longueurs des arêtes de $G$. Dans ce paragraphe, nous donnons des résultats, dont certains sont nouveaux, sur cette correspondance. Remarquons tout d'abord que, pour un graphe d'arches $G$ sur $X$ donné, il existe en 
général plusieurs ordres conformes à la condition (OEI). Il en est ainsi, pour le graphe d'arches de la figure 1, de l'ordre a, b, c, f, d, e, et de plusieurs autres. Il y a donc lieu d'examiner si le résultat dépend du choix d'un tel ordre. En fait, un résultat présenté dans [38] dans le cas particulier des 2-arbres s'étend à tous les graphes d'arches.

THÉORÈME 5.1 [26]. La méthode des triangles associe de façon unique, indépendante de l'ordre de parcours des sommets, une fonction d'arbre à un graphe d'arches valué.

Cependant, il peut exister d'autres fonctions d'arbres préservant les longueurs des arêtes de $G$ que celle obtenue par la méthode des triangles, comme le montre l'exemple du graphe d'arches valué la figure 6, à gauche [25]. Après avoir déterminé la 3 -étoile de feuilles $\mathrm{a}, \mathrm{b}$ et $\mathrm{c}$, on a une infinité de positions possibles pour $\mathrm{d}$ qui toutes correspondent à la longueur 5 pour les chemins entre $a$ et $d$ et entre $b$ et $d$ dans l'arbre. La figure 6, à droite, montre quatre de ces positions, d1 étant celle fixée par la méthode des triangles.
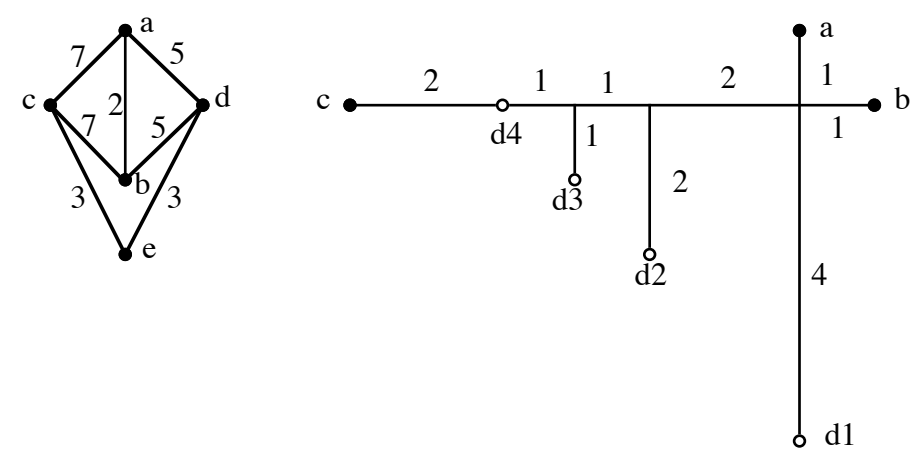

Figure 6. Plusieurs positions pour le sommet d

THÉORÈME 5.2 [26]. Pour un graphe d'arches valué $G$, les deux conditions suivantes sont équivalentes :

(i) la seule fonction d'arbre préservant les longueurs des arêtes de $G$ est celle donnée par la méthode des triangles;

(ii) le FEX-arbre obtenu par la méthode des triangles est complètement résolu.

Examinons maintenant la question d'inversibilité suivante : étant donnée une distance d'arbre $d$ et un graphe d'arches $G=(X, E)$, valuons les arêtes de $G$ selon les valeurs correspondantes de $d$, puis appliquons la méthode des triangles au graphe d'arches valué ainsi obtenu. On en déduit une fonction d'arbre $d^{\prime}=d^{\prime}{ }_{G, d^{\prime}}$. À quelles conditions sur $G$ retrouve-t-on la distance initiale $d$ ? Notons $\mathcal{G}(d)$ l'ensemble des graphes d'arches pour lesquels on a $d=d^{\prime}, \mathcal{H}(d)$ celui des éléments de $\mathcal{G}(d)$ qui sont des 2-arbres, et $\mathcal{H}$ l'ensemble des 2-arbres sur $X$.

Il a été montré dans [38] que l'ensemble $\mathcal{H}(d)$, et donc $\mathcal{G}(d)$ qui le contient, n'est pas vide. Par conséquent, à partir du théorème 5.2, on a : 
COROLLAIRE 5.3 Soient $d$ une distance d'arbre et $G$ un graphe d'arches sur $X$, valué selon $d$. Si le FEX-arbre obtenu par la méthode des triangles est résolu, alors $G \square \mathcal{G}(d)$.

Le résultat suivant étend à $\mathcal{G}(d)$ une caractérisation des éléments de $\mathcal{H}(d)$ donnée dans [38].

THÉORÈME 5.4 Pour une distance d'arbre $d$ et un graphe d'arches $G$ sur $X$, les trois conditions suivantes sont équivalentes :

(i) $\quad G \square \mathcal{G}(d)$;

(ii) Il y a un ordre sur $X$ conforme à la condition (OEI) tel que, pour $i=3, \ldots, n$, le triplet $\left(y, x_{i}, z\right)$ est bien formé dans $T^{i}$;

(iii) Pour tout ordre sur $X$ conforme à la condition (OEI) et pour $i=3, \ldots, n$, le triplet $\left(y, x_{i}, z\right)$ est bien formé dans $T^{i}$;

Preuve. Il est évident que (iii) implique (ii). Si (ii) est vraie, on retrouve exactement le FEX-arbre représentant $d$ quand on applique, en suivant l'ordre spécifié par (ii), la méthode des triangles au graphe d'arches $G$ valué selon $d$, d'où $d^{\prime}=d_{G, d^{\prime}}$. Donc, (ii) implique (i).

Il reste à montrer que (i) implique (iii). Choisissons un ordre sur $X$ conforme à la condition (OEI), et considérons la 2-feuille $x_{i}$ du graphe d'arches $G^{i}$ induit par les sommets $x_{1}, x_{2}, \ldots, x_{i}$ de $G$. Elle appartient à une unique arche $\left\{x_{i}, y, z\right\}$ de $G^{i}$. Considérons la restriction $d_{i-1}$ de $d$ à $\left\{x_{1}, x_{2}, \ldots, x_{i-1}\right\}$. Par construction, le graphe d'arches $G^{i-1}$ induit par les sommets $x_{1}, x_{2}, \ldots, x_{i-1}$ de $G$ est un élément de $\mathcal{G}\left(d_{i-1}\right)$. Nous pouvons alors lui substituer un 2-arbre $H^{i_{-1}}$, élément de $\mathcal{H}\left(d_{i-1}\right)$ et tel que $y z$ en est une arête (selon le corollaire 4.5 de [38], un tel 2-arbre existe toujours). On obtient ensuite un 2-arbre $H^{i}$ sur $\left\{x_{1}, x_{2}, \ldots, x_{i}\right\}$ en ajoutant le sommet $x_{i}$ et les arêtes $x_{i} y$ et $x_{i} z$ au 2-arbre $H^{i-1}$. Alors, la $i$-ème étape de la méthode des triangles appliquée à $H^{i}$ est complètement identique à celle appliquée à $G^{i}$. On en déduit, d'une part, que $H^{i}$ appartient à $\mathcal{H}\left(d_{i}\right)$, et, d'autre part, comme conséquence du théorème 4.2 de [38], que le triplet $\left(y, x_{i}, z\right)$ est bien formé dans $T^{i}$.

Il a été rappelé dans l'introduction que plusieurs façons de résumer une distance d'arbre par 2n-3 de ses entrées ont été décrites dans la littérature. Ces résumés répertoriés correspondent tous en fait à des éléments de $\mathcal{H}(d)$ [38], à commencer par les codages d'arbres proposés par Chaiken, Dewdney et Slater [16] et par Yushmanov [52] ; cf. [43]. Ceci constitue une première indication sur le rôle particulier des 2-arbres. De plus, il y a des résultats spécifiques sur les liens entre un 2-arbre valué $G$ et le FEXarbre $T$ lui correspondant par la méthode des triangles. Ainsi, une arête $e$ d'un 2-arbre $G$ appartient à au moins un triangle de $G$; soit alors $\partial(e)$ le nombre de triangles de $G$ contenant $e$. On montre dans [38] que la quantité $\square(G, d)=\sum_{e \square E}(2-\partial(e)) d(e)$ est égale à deux fois la longueur totale du FEX-arbre $T$. On a ensuite la caractérisation suivante des éléments de $\mathcal{H}(d)$, dont l'extension aux graphes d'arches nécessiterait, au préalable, celle du paramètre $\square$ : 
THÉORÈME 5.5 [38]. Pour une distance d'arbre $d$ et un 2-arbre $G$ sur $X$, les deux conditions suivantes sont équivalentes :

(i) $\quad G \square \mathcal{H}(d)$;

(ii) $\square(G, d)=\min \left\{\square\left(G^{\prime}, d\right): G^{\prime} \square \mathcal{H}\right\}$;

Ainsi, les éléments de $\mathcal{H}(\mathrm{d})$ prolongent, pour les distances d'arbre, une partie des propriétés des arbres minimums pour les ultramétriques :

- la minimalité de la longueur des arbres minimums est remplacée par celle du paramètre $\square$.

- les $n$-1 valeurs des arêtes de l'arbre minimum résument les $n(n-1) / 2$ entrées d'une ultramétrique ; de même, les $2 n-3$ valeurs des arêtes d'un élément de $\mathcal{H}(d)$ résument les entrées d'une distance d'arbre.

Pour les graphes d'arches, éléments de $\mathcal{G}(d)$, on peut simplement dire qu'ils minimisent la longueur du FEX-arbre obtenu par la méthode des triangles. Enfin, il ne faut sans doute pas espérer trouver aux éléments de $\mathcal{G}(d)$ ou de $\mathcal{H}(d)$ autant de propriétés qu'aux arbres minimums, car, comme il a été observé au paragraphe 3 avec l'absence de la propriété d'échange, les ensembles des graphes d'arches et des 2-arbres sur $X$ n'ont pas les fortes propriétés structurelles de celui des arbres sur $X$.

\section{CONCLUSION}

Nous avons d'abord présenté la méthode des triangles, qui a constitué la motivation initiale de notre intérêt pour les graphes d'arches. Nous avons fait une revue commentée (et certainement encore incomplète) de ces graphes dans les paragraphes 3 et 4 . Enfin, dans le paragraphe 5, nous reprenons la correspondance entre graphes d'arches et FEXarbres valués induite par la méthode des triangles, et prolongeons aux graphes d'arches des résultats précédemment montrés dans le cas des 2-arbres.

On a vu au paragraphe 3.3 (proposition 3.1) que le rapprochement des graphes d'arches et des 2-arbres a des conséquences intéressantes. Il suscite aussi naturellement des problèmes comme : les graphes d'arches constituent-ils une classe de graphes de largeur en arbre bornée ? On a en effet vu qu'un tel graphe peut contenir un 2-cycle et n'est alors pas de largeur arborescente 2. Du côté de l'analyse des distances et dissimilarités, une première question est l'étude des distances définies à partir des 2arbres par Proskurowski ([46] ; voir la fin de la section 4.2).

Une autre direction de recherches est inspirée par une thèse récente [13]. Considérons une relation symétrique $R$ sur $X$, et l'opération suivante : si une partie $Y$ de $X$ induit une $k$-clique privée d'exactement une paire $y y^{\prime}$ d'éléments de $Y$, on ajoute la paire $y y^{\prime}$ à $R$. En recommençant cette opération tant qu'il est possible, on obtient une relation $\hat{R}$ qui, pour $k=3$, n'est autre que la fermeture transitive usuelle de $R$. C'est pourquoi nous dirons que $\hat{R}$ est la fermeture $k$-transitive de $R$. On sait que la relation $R$ engendre l'ensemble de toutes les paires d'éléments de $X$ par 
fermeture transitive si et seulement si elle contient un arbre sur $X$. On voit facilement que, de même, elle engendre l'ensemble de toutes les paires d'éléments de $X$ par fermeture $k$-transitive si elle contient un graphe de $k$-arches sur $X$. Cette remarque rend compte de l'intérêt des graphes d'arches pour l'étude de certaines classes de dissimilarités définies par une condition sur quatre points, comme le sont les dissimilarités d'arbres. On peut alors étendre cette relation à d'autres types de dissimilarités, et d'autres valeurs de $k$. Des classes de dissimilarités définies par une condition sur $k$ points, comme les $k$-ultramétriques de Jardine et Sibson [30], sont reconnues dans [13].

Remerciements : L'auteur tient à remercier Alain Guénoche pour nos fructueuses discussions, et pour le terme "graphes d'arches" lui-même, ainsi que les deux rapporteurs anonymes pour leurs corrections, remarques, références, et généralement pour leur lecture attentive.

\section{BIBLIOGRAPHIE}

[1] AIGNER M., Combinatorial Theory, Berlin, Springer-Verlag, 1979.

[2] ARNBORG S., CORNEIL D.G., PROSKUROWSKI A., " Complexity of finding embeddings in a $k$-tree ", SIAM J. Alg. Disc. Meth. 8, 1987, p. 277-284.

[3] ARNBORG S., PROSKUROWSKI A., " Characterization and recognition of partial $k$-trees ", Congr. Numer. 47, 1985, p. 69-75.

[4] ARNBORG S., PROSKUROWSKI A., " Characterization and recognition of partial 3-trees ”, SIAM J. Alg. Disc. Meth. 7, 1986, p. 305-314.

[5] ARNBORG S., PROSKUROWSKI A., " Linear time algorithms for NP-hard problems restricted to partial $k$-trees ", Discrete Appl. Math. 23, 1989, p. 11-24.

[6] BARTHÉLEMY J.P., GUÉNOCHE A., Les arbres et les représentations des proximités, Paris, Masson, 1988.

[7] BEINEKE L.W., PIPPERT R.E., “ The number of labelled $k$-dimensional trees ”, J. Comb. Theory 6, 1969, p. 200-205.

[8] BERGE C., Graphes et hypergraphes, Paris, Dunod, 1970.

[9] BODLAENDER H.L., " Treewidth: algorithmic techniques and results", Proceedings $22^{\text {nd }}$ International Symposium on Mathematical Foundations of Computer Sciences, MFCS'97, I. Privara, P. Ruzicka, eds., Lecture Notes in Computer Sciences 1295, Berlin, Springer-Verlag, 1997, p. 19-36.

[10] BONDY J.A., “ Trigraphs ”, Discrete Math. 75, 1989, p. 69-79.

[11] BORIE R.B., PARKER R.G., TOVEY C.A., " Deterministic decomposition of recursive graph classes ", SIAM J. Disc. Math. 4, 1991, p. 481-501.

[12] V. BOUCHITTÉ, I. TODINCA, “ Treewidth and minimum fill-in: grouping the minimal separators ”, SIAM J. on Computing, 2002, [à paraître]. 
[13] BRUCKER F., " Modèles de classification en classes empiétantes ", Thèse de l'École des hautes études en sciences sociales, Paris, 2001.

[14] BUNEMAN P., " The recovery of trees from measures of dissimilarity", Mathematics in Archaeological and Historical Sciences, F.H. Hodson, D.G. Kendall, P. Tautu, eds., Edimburg, Edimburg University Press, 1971, p. 387-395.

[15] BUNEMAN P., “ A characterization of rigid circuit graphs ”, Discrete Math. 9, 1974, p. 205-212.

[16] CHAIKEN S., DEWDNEY A.K., SLATER P.J., “ An optimal diagonal treecode ", SIAM J. Alg. Disc. Math. 4, 1983, p. 42-49.

[17] COURCELLE B., “ The monadic second-order logic of graphs I: recognizable sets of finite graphs ", Information and Computation 85, 1990, p. 12-65.

[18] DEWDNEY A.K., “ Higher dimensional tree structures ”, J. Comb. Theory B 17, 1974, p. 160-169.

[19] DIRAC G.A., “ A property of 4-chromatic graphs and some remarks on critical graphs ”, J. London Math. Soc. 27, 1952, p. 85-92.

[20] FARLEY A.M., “Networks immune to isolated failures ”, Networks 11, 1981, p. 255-268.

[21] FOWLER T., GESSEL I., LABELLE G, LEROUX P., " The specification of 2trees ", Advances in Applied Mathematics, 2001, [à paraître].

[22] FREUDER E.C., “Complexity of K-Tree Structured Constraint Satisfaction Problems ", Proceedings of the $8^{\text {th }}$ National Conference on Artificial Intelligence, AAAI Press / The MIT Press, 1990, p. 4-9.

[23] GOLUMBIC M.C., Algorithmic graph theory and perfect graphs, New York, Academic Press, 1980.

[24] GRANOT D., SKORIN-KAPOFF D., “ NC algorithms for recognizing partial 2trees and 3-trees ", SIAM J. Disc. Math. 4, 1991, p. 342-354.

[25] GUÉNOCHE A., LECLERC B., “ The triangles method to build X-trees from incomplete distance matrices ", RAIRO Operations Research, 2001, [à paraître].

[26] GUÉNOCHE A., LECLERCB., MAKARENKOV V., " On the extension of a partial metric to a tree metric ", Les cahiers du CAMS n ${ }^{\circ} 195$, septembre 2000, 18 pages.

[27] HARARY F., Graph theory, Reading, Mass., Addison-Wesley, 1969.

[28] HARARY F., PALMER E.M., “ On acyclical simplicial complexes ”, Mathematika 15, 1968, p. 115-122.

[29] IDURY R.M., SCHÄFFER A.A., “ Triangulating three-colored graphs in linear time and linear spaces ", SIAM J. Disc. Math. 6, n 2, 1993, p. 289-293.

[30] JARDINE N., SIBSON R., Mathematical Taxonomy, Londres, Wiley, 1971.

[31] KANNAN S.K., WARNOW T.J., “ Triangulating 3-coloured graphs ", SIAM J. Discrete Math. 5, $\mathrm{n}^{\circ}$ 2, 1992, p. 249-258.

[32] KLOKS T., Treewidth: computations and approximations, Lecture notes in computer science 842, Berlin, Springer, 1994. 
[33] KURATOWSKI K., " Sur le problème des courbes gauches en topologie ", Fundamenta Mathematicae 15, 1930, p. 271-283.

[34] LECLERC B., “ Description combinatoire des ultramétriques ”, Math. Sci. hum. 73, 1981, p. 5-37.

[35] LECLERCB., " Adjusments of tree metrics based on minimum spanning trees ", New Approaches in Classification and Data Analysis, E. Diday et al., eds., Berlin, Springer-Verlag, 1994, p. 128-135.

[36] LECLERC B., “ Minimum spanning trees for tree metrics: abridgements and adjustments" J. of Classification 12, 1995, p. 207-241.

[37] LECLERC B., “ Minimum spanning trees and types of dissimilarities ”, European Journal of Combinatorics 17, $\mathrm{n}^{\circ} 2 / 3,1996$, p. 255-264.

[38] LECLERC B., MAKARENKOV V., " On some relations between 2-trees and tree metrics ”, Discrete Math. 192, 1998, p. 223-249.

[39] LIU P.C., GELDMACHER R.C., “ An $O(\max (m, n))$ algorithm for finding a subgraph homeomorphic to $K_{4}$ ", Proceedings $11^{\text {th }}$ Southeastern Conference on Combinatorics, Graph Theory and Computing, 1980, p. 597-609.

[40] McKEE T.A., McMORRIS F.R., Topics in intersection graph theory, Philadelphie, SIAM, 1999.

[41] MCMORRIS F.R., WARNOW T.J., WIMER T., “ Triangulating vertex-colored graphs ", SIAM J. Disc. Math. 7, n 2, 1994, p. 296-306.

[42] MAKARENKOV V., Propriétés combinatoires des distances d'arbre : algorithmes et applications, Paris, Thèse de l'EHESS, 1997.

[43] MAKARENKOV V., LECLERC B., "Circular orders of tree metrics, and their uses for the reconstruction and fitting of phylogenetic trees ", Mathematical hierarchies and Biology, B. Mirkin, F.R. McMorris, F. Roberts, A. Rzhetsky, eds., DIMACS Series in Discrete Mathematics and Theoretical Computer Science, Amer. Math. Society, 1997, p. 183-208.

[44] MOON J.W., “ The number of labelled $k$-trees ”, J. Comb. Theory 6, n 2, 1969, p. 196-199.

[45] PIPPERT R.E., BEINEKEL.W., " Characterisation of 2-dimentional trees ”, The Many Facets of Graph Theory, G. Chatrand, SF. Kapoor, eds., Lecture Notes in Mathematics 110, Berlin, Springer-Verlag, 1969, p. 263-270.

[46] PROSKUROWSKI A., " Separating subgraphs in $k$-trees: cables and caterpillars ", Discrete Math. 49, 1984, p. 275-295.

[47] ROSE D.J., “ On simple characterizations of $k$-trees ”, Discrete Math. 7, 1974, p. 317-322.

[48] TODD P., " A $k$-tree generalization that characterizes consistency of dimensioned engineering drawings ”, SIAM J. Disc. Math. 2, n 2, 1989, p. 255-261.

[49] VAN LEUWEN J., Ch. 10, “ Graph algorithms ", Handbook of Computer Science, vol A. Algorithms and complexity, J. Van Leuwen, ed., Amsterdam, Elsevier, 1990, p. 525-631. 
[50] WALD A., COLBOURN C.J., " Steiner trees, partial 2-trees and minimum IFI networks ", Networks 13, 1983, p. 159-167.

[51] WELSH D.J.A., Matroid Theory, Londres, Academic Press, 1976.

[52] YUSHMANOV S.V., " Construction d'un arbre à $p$ feuilles à partir de 2p-3 éléments de sa matrice de distance ", (en russe), Matematicheskie Zametki, 35, 1984, p. 877-887. 


\section{ANNEXES}

Les graphes d'arches non étiquetés à $n$ sommets, $4 \leq n \leq 6$.

$n=4$

$n=5$
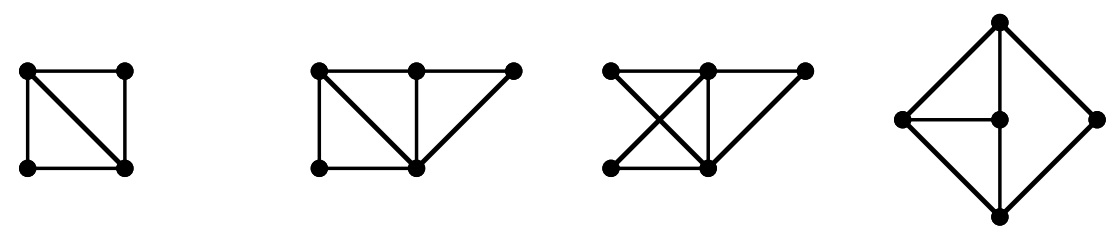

$n=6$
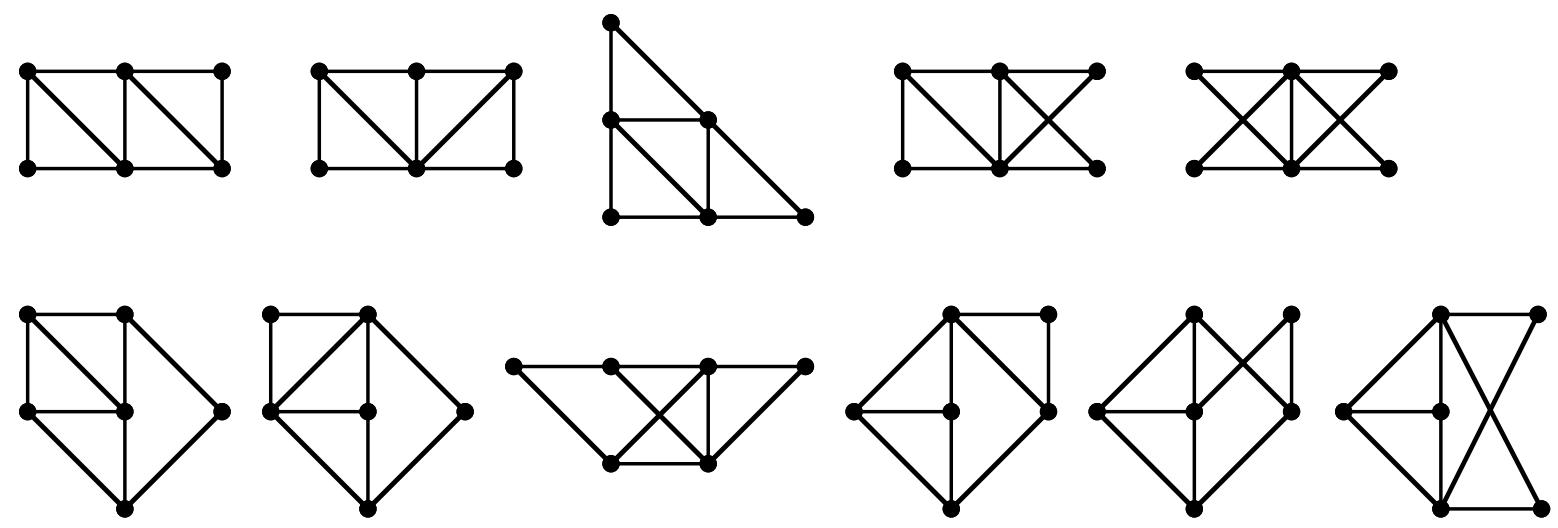

Les 2-arbres non étiquetés à $n$ sommets, $n \leq 8$.

$n=7$
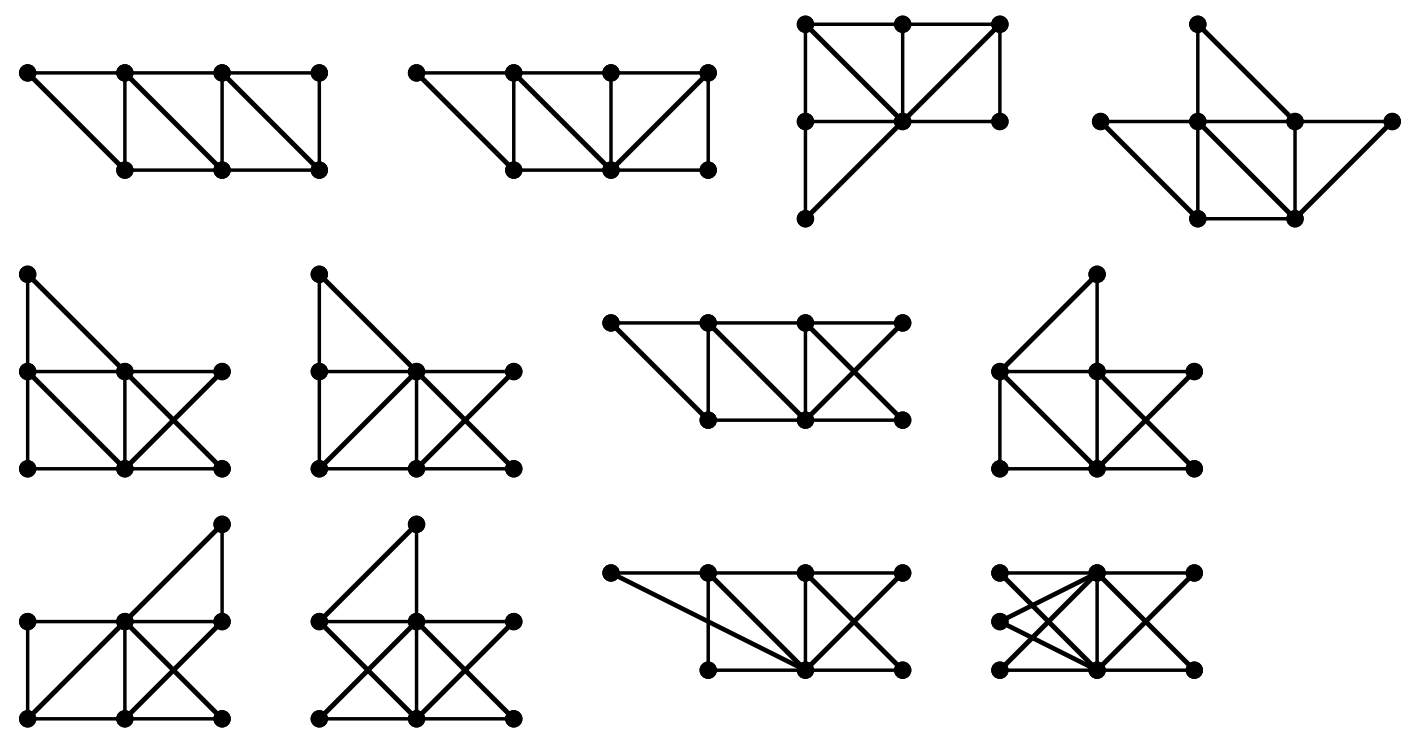
$n=8$
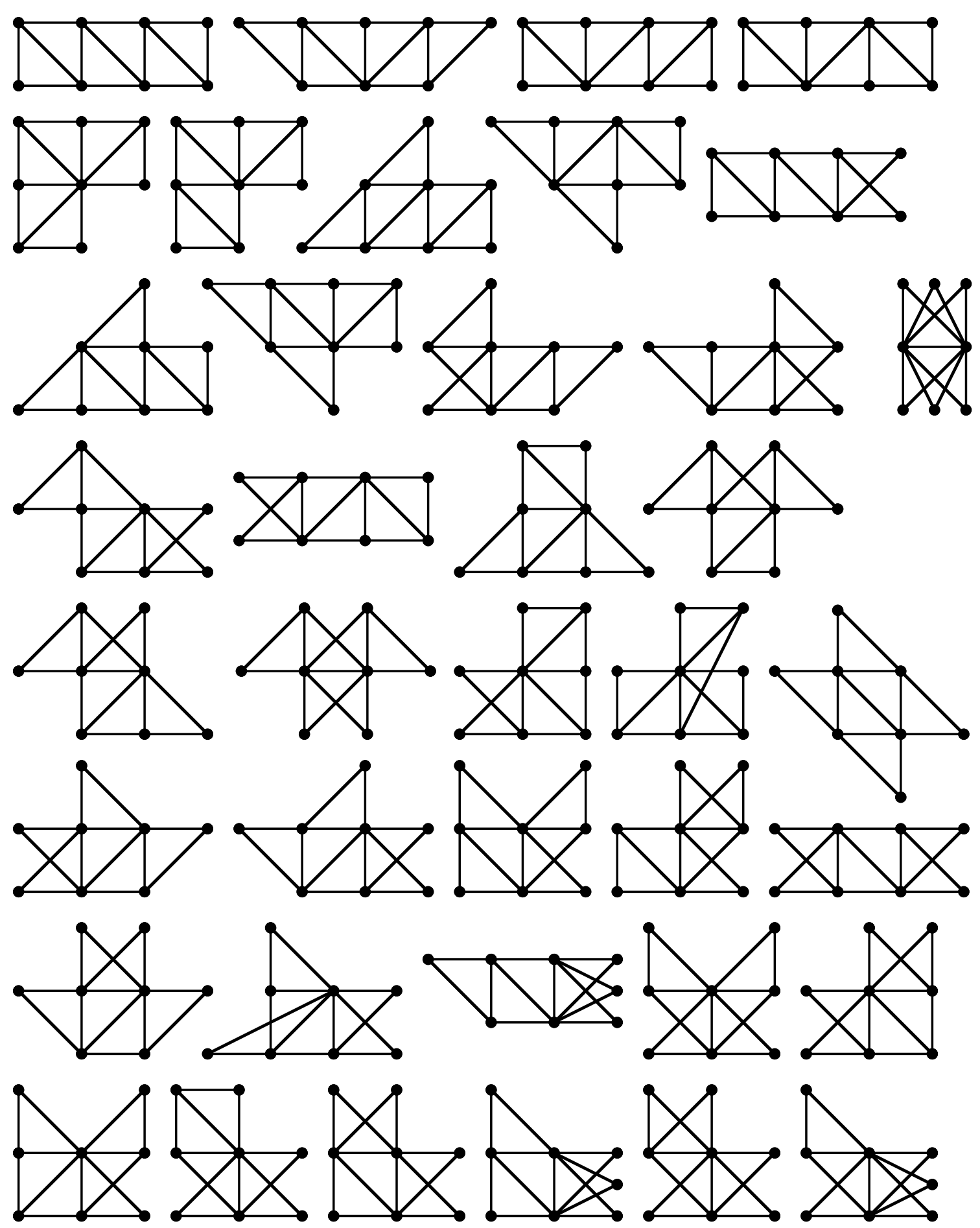